\section{The Pesticide}

\section{Properties DataBase}

\author{
by Kathy Lewis and Andy Green
}

M odeling and decision support systems for pesticide risk assessments are invariably data hungry, and there has been a long and widely recognized need for a single authoritative and comprehensive database of pesticide physicochemical and toxicological properties that is available both as an online resource and in a portable electronic format suitable for embedding in or interrogating from other software applications. The Pesticide Properties DataBase (PPDB) has been developed to address this need. The online system is free of charge and currently contains records for about 1800 active substances and their metabolites. It holds data required for modeling the fate and ecotoxicological risks of pesticides in both terrestrial and aquatic systems, as well as information on chemical identifiers and data relating to regulatory issues and commercial products.

\section{Database Design and Description}

The database has been designed to meet two primary objectives. First, it aims to provide a single, comprehensive source of reliable, consistently presented pesticide data having common syntax, units, and semantics. Second, it seeks to ensure that the information is accessible to and usable by as wide a range of end users as possible.

During database development two distinct end-user types were identified: first, users who require large

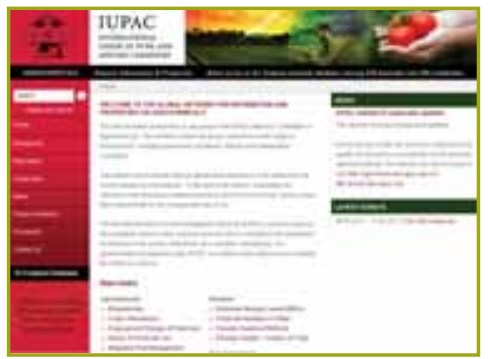

general information: e.g., various identifier names, codes and reference numbers, language translations, chemical group, formula, structures, and country registration data

- physicochemical data: e.g., solubility, vapor pressure, density, refractive index, and dissociation constants

- environmental fate: e.g., the octanol-water partition constant, Henry's law constant, degradation rates in soil, sediments, and water, the Freundlich coefficient, the organic-carbon sorption constant, and information on degradation products

- human health: e.g., WHO toxicity classifications, Acceptable Daily Intakes, toxicity to mammals, other exposure limits and toxicity endpoints, recognized health issues plus the EC risk and safety classifications

- ecotoxicology: e.g., acute and chronic toxicity data for a wide range of fauna and flora plus information on bioaccumulation

In addition to "basic" data, a range of "metadata" is also provided. For example, "layperson" interpretations of the data are presented by applying classification thresholds, such as those used to indicate a need for detailed risk assessment under European Directive $91 / 414$, or "rules of thumb" in general use, such as the guidelines used by the UK pesticide industry for developing Pesticide Environmental Information Sheets. ${ }^{1}$ The online system also automatically calculates a number of "coarse" indicators, such as the GUS Index for groundwater leaching potential 2 and a measure of particle-bound transport that indicates the pesticide's risk of being transported with runoff. 3

A number of support facilities are data sets for software applications such as risk assessments, and second, users seeking particular parameters for a specific chemical. To satisfy the needs of both, the approach adopted was to store the data using MS Access 2000 and then stream this through various "data filters" for formatting into HTML pages for online access. It was determined that the MS Access format (or an MS Excel export) would probably meet the needs of most software applications, whereas the online version would be the ideal way to deliver data for most other uses. This approach also helps simplify the updating and maintenance process.

Data stored can be divided into a number of discrete areas: also available online, including extensive documentation, a comprehensive search facility, an A-Z index, print facilities, and an e-mail help line.

\section{Database Population}

Probably the best sources of information currently available for pesticide properties are the monographs produced as part of regulatory processes such as the EU 91/414 review, and data within these types of documents have been the first choice for populating the database. Where regulatory monographs are not available, alternative sources are used, including data provided by regulatory authorities, charities, NGOs, 
peer-reviewed journals, research, manufacturers, and online and hard-copy data publications.

As the primary purpose of the PPDB is risk assessment, data have been selected for this particular use. The values quoted for physicochemical and fate properties are usually a mean of the various studies identified. Where data are particularly sensitive, to climate or soil, for example, information on the data range has been added. Where data are just naturally very variable, the most appropriate for EU conditions have been selected. For toxicological data, "worstcase" values have been selected. The data relate to, wherever possible, specific species and endpoints to ensure a harmonized and balanced data set.

\section{Database Quality Assurance}

Accuracy of the data always challenges the accuracy and reliability of risk assessment processes, as the results will only ever be as good as the data driving them. Pesticide data are inherently variable, and sources are not always as reliable as would be liked. Consequently the PPDB uses a unique "Quality Barometer"- a code tagged to most parameters that reflects the data source and the extent to which the data are considered reliable and verifiable.

The Quality Barometer consists of two parts. The first is used to identify the type of data source (e.g., regulatory, peer-reviewed publication, manufacturer's data, etc.). The second part is a confidence score in the range $0-5$. These scores are awarded based on a set of rules and reflect confidence in the source. For example, a regulatory dossier would usually be awarded a 5, whereas data from a nonstandard and unreferenced source may be awarded a 1 or 0 . It should be emphasized that a low score does not necessarily mean poor quality data, just that the data have not be verified.

Accuracy and reliability of the data are not the only data quality issue. Database management is conducted according to strict protocols, and data are reviewed and updated on a continuous and ongoing basis.

\section{Database Access and Usage}

The online version was launched on a free-to-all basis in late 2006, and the IUPAC specific portal was released about a year later. Access has been monitored using an embedded web analysis tool, and, as shown in the chart, usage has grown from year to year. Around 42 percent of access is from within Europe, and a further 30 percent from the USA and Canada. The online database has had about 750000 visitors to date.

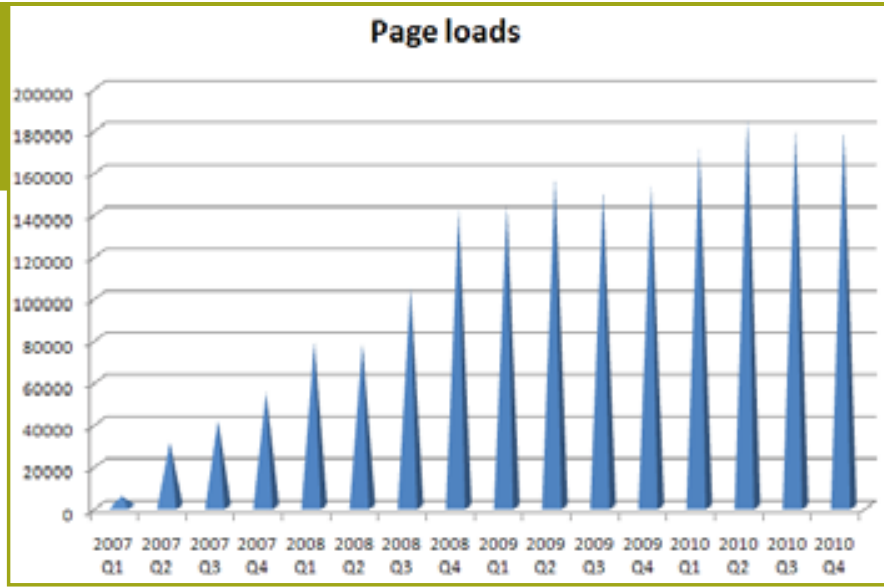

PPDB data have been used to support a variety of applications. These include the EU 6th Framework Programme FOOTPRINT project, which developed a suite of pesticide aquatic risk and management tools; 4 the EU Life project ArtWet, which developed a model to predict pesticide pollution in surface waters following runoff events; 5 and the development of a pesticide risk indicator for Tasmania. ${ }^{6}$ PPDB data also support the online ASTERisk system, ${ }^{7}$ which is a tool to support the sustainable use of pesticides in the Lombardy region of Italy.

\section{References}

1. The Voluntary Initiative Pesticide Information Sheets, available at http://www.voluntaryinitiative.org.uk/content/eis.aspx.

2. D.I. Gustafson, "Groundwater Ubiquity Score: A Simple Method for Assessing Pesticide Leachability," Environmental Toxicology and Chemistry 8 (1989): 339357.

3. D. Goss and R.D. Wauchope, "The SCR/ARS/CES Pesticides Properties Database: II; Using It with Soils Data in a Screening Procedure," In D.L. Weigmann, ed., Pesticides in the Next Decade: The Challenge Ahead (Blacksburg, VA: Virginia Water Resources Research Center, 1990), 471-493.

4. A. Green, K.A. Lewis, and J. Tzilivakis, "FOOTPRINT: Creating Tools for Pesticide Risk Assessment and Management in Europe," AAB Conference: Pollution from Pesticides-Point Source versus Diffuse, Harper Adams University, Newport, 25 November 2008.

5. D. Elsaesser and R. Schulz, "The ArtWET Tool: A Georeferenced Approach Assessing Runoff-Related Pesticide Pollution in Surface Waters across Europe," Applied Geoinformatics for Society and Environment, 3rd International Summer School and Conference Proceedings 109 (2010): 69-74.

6. R. Kookana and R. Correll, The Tasmanian River Catchment Water Quality Initiative Pesticide Impact Rating Index (PIRI), Risk Indicator for Minimising Off-Site Migration of Pesticides, CSIRO Land and Water Science Report 30/08 (2008).

7. International Centre for Pesticides and Health Risk Prevention (ICPS), The ASTERisk tool. Available at http:// www.icps.it/ixps/AuthFiles/login.asp.

\section{http://pesticides.iupac.org}

